\title{
Towards quantitative simulations of high power proton cyclotrons
}

\author{
Y. J. Bi, ${ }^{1,2,3, *}$ A. Adelmann, ${ }^{2, \dagger}$ R. Dölling, ${ }^{2}$ M. Humbel, ${ }^{2}$ W. Joho, ${ }^{2}$ M. Seidel, ${ }^{2}$ and T. J. Zhang ${ }^{1}$ \\ ${ }^{1}$ China Institute of Atomic Energy, Beijing, 102413, China \\ ${ }^{2}$ Paul Scherrer Institut, Villigen, $\mathrm{CH}-5232$, Switzerland \\ ${ }^{3}$ Department of Engineering Physics, Tsinghua University, Beijing, 100084, China
}

(Received 23 December 2010; published 23 May 2011)

\begin{abstract}
We describe a large scale simulation effort using Object Oriented Parallel Accelerator Library, that leads to a better quantitative understanding of the existing Paul Scherrer Institut high power proton cyclotron facility. The 1.3 MW of beam power on target poses stringent constraints on the controlled and uncontrolled beam losses. We present initial conditions for the Ring simulation, obtained from the new time-structure measurement and the many profile monitors of the $72 \mathrm{MeV}$ transfer line. A trim coil model is developed, including trim coil TC15, which is needed to avoid the dangerous $\nu_{r}=2 \nu_{z}$ resonance. By properly selecting the injection position and angle (eccentric injection), the flattop voltage, and phase, very good agreement between simulations and measurements at the radial probe RRE4 is obtained. We report on 3-4 orders of magnitude in dynamic range when comparing simulations with measurements. The relation between beam intensity, rms beam size, and accelerating voltage is studied and compared with measurement. The demonstrated capabilities are mandatory in the design and operation of the next generation high power proton drivers. In an outlook we discuss our future plans to include more physics into the model, which eventually leads to an even larger dynamic range in the simulation.
\end{abstract}

DOI: 10.1103/PhysRevSTAB.14.054402

PACS numbers: 29.20.dg

\section{INTRODUCTION}

Paul Scherrer Institut (PSI) operates a cyclotron based high intensity proton accelerator routinely at an average beam power of $1.3 \mathrm{MW}$. With this power the facility is at the worldwide forefront of high intensity proton accelerators. An upgrade program is under way to ensure high operational reliability and push the intensity to even higher levels. The beam current is limited in practice by losses at extraction and the resulting activation of accelerator components. Further intensity upgrades and new projects aiming at an even higher average beam power are only possible if the relative losses can be lowered in proportion, thus keeping absolute losses at a constant level.

Maintaining beam losses at levels allowing hands-on maintenance is a primary challenge in any high power proton machine design and operation. For a 1.3 MW beam in the PSI Ring cyclotron, this corresponds to a transmission of $99.97 \%$ taking controlled and uncontrolled losses into account. In a $10 \mathrm{MW}$ class machine we require the losses to be on the same level which is a challenging task and is asking for precise beam dynamics calculation. In consequence, predicting beam halo at these levels is a great challenge and will be addressed in this paper.

\footnotetext{
*biyj05@mails.tsinghua.edu.cn

${ }^{\dagger}$ andreas.adelmann@psi.ch
}

Published by the American Physical Society under the terms of the Creative Commons Attribution 3.0 License. Further distribution of this work must maintain attribution to the author(s) and the published article's title, journal citation, and DOI.
High power hadron drivers have being used in many disciplines of science and a growing interest in cyclotron technology for high power hadron drivers has been shown very recently. Two very recent papers demonstrate this fact: (1) The search for $C P$ violation in the neutrino sector [1] calls ultimately for three machines in the megawatt range at an energy of $800 \mathrm{MeV}$. (2) In [2], a white paper on "Accelerator and Target Technology for Accelerator Driven Transmutation and Energy Production," the cyclotron technology is advertised. We quote: "On the whole, the development status of accelerators is well advanced, and beam powers of up to 10 MW for cyclotrons and 100 MW for linacs now appear to be feasible...".

This report will briefly introduce Object Oriented Parallel Accelerator Library (OPAL), a tool for precise beam dynamics simulations including 3D space charge. One of OPAL's "flavors" (OPAL-cycl) is dedicated to high power cyclotron modeling and is explained in greater detail. We then explain how to obtain initial conditions for our PSI Ring cyclotron which still delivers the world record in beam power of $1.3 \mathrm{MW}$ in continuous wave (cw) operation. Several crucial steps are explained, necessary to be able to predict tails at the level of $3 \sigma-4 \sigma$ in the PSI Ring cyclotron. We compare our results at the extraction with measurements, obtained with a $1.18 \mathrm{MW} \mathrm{cw}$ production beam. Based on measurement data, we develop a simple linear model to predict beam sizes of the extracted beam as a function of intensity and confirm the model with simulations. A conclusion and discussions to include more physics into the model, which eventually 
leads to a even larger dynamic range in the simulation, closes the paper.

\section{BASIC EQUATIONS AND PHYSICAL MODEL A. A BRIEF INTRODUCTION TO OPAL}

OPAL is a tool for charged-particle optic calculations in large accelerator structures and beam lines including 3D space charge. OPAL is built from first principles as a parallel application; it admits simulations of any scale, on the laptop and up to the largest high performance computing (HPC) clusters available today. Simulations, in particular HPC simulations form the third pillar of science, complementing theory and experiment. OPAL includes various beam line element descriptions and methods for single particle optics, namely maps up to arbitrary order, symplectic integration schemes, and lastly time integration [3]. OPAL is based on the independent parallel particle layer (IPPL) [4] which adds parallel capabilities. Main functions inherited from IPPL are structured rectangular grids, fields and parallel fast Fourier transform (FFT), and particles with the respective interpolation operators. Recently, we added a powerful iterative solver to OPAL taking into account complicated boundary conditions [5]. More details on cyclotron modeling which are direct relevant to this article can be found in [6]. Several flavors of OPAL are available; for details we refer to the User Manual [3]. In this paper we use OPAL-t for the tracking of $72 \mathrm{MeV}$ beam line, connecting two cyclotrons, Injector 2 and the Ring cyclotron. The other OPAL flavor-OPALcycl-is designed specially for cyclotron beam dynamics and is explained in the next section.

\section{B. THE BEAM DYNAMICS MODEL OF OPAL-cycl}

In the cyclotrons and beam lines under consideration, the collisions between beam particles can be neglected because the typical bunch densities are low. In the time domain, the general equations of motion of a charged particle in electromagnetic fields can be expressed by

$$
\frac{d \mathbf{p}(t)}{d t}=q(c \boldsymbol{\beta} \times \mathbf{B}+\mathbf{E}),
$$

where $m_{0}, q$, and $\gamma$ are rest mass, charge, and the relativistic factor. With $\mathbf{p}=m_{0} c \gamma \boldsymbol{\beta}$ we denote the momentum of a particle, $c$ is the speed of light, and $\boldsymbol{\beta}=\left(\beta_{x}, \beta_{y}, \beta_{z}\right)$ is the normalized velocity vector. In general the time $(t)$ and position (x) dependent electric and magnetic vector fields are written in abbreviated form as $\mathbf{B}$ and $\mathbf{E}$, where $\mathbf{E}$ and $\mathbf{B}$ include both external applied fields and space charge fields:

$$
\mathbf{E}=\mathbf{E}_{\mathrm{ext}}+\mathbf{E}_{\mathrm{sc}}, \quad \mathbf{B}=\mathbf{B}_{\mathrm{ext}}+\mathbf{B}_{\mathrm{sc}} .
$$

The model of the external magnetic field is based on midplane field measurements with excited trim coils. In consequence, we have a vertical field, $B_{z}$, measured on the median plane $(z=0)$ as a function of azimuthal angle $(\theta)$.
Since the magnetic field outside the median plane is required to compute trajectories with $z \neq 0$, the field needs to be expanded in the $Z$ direction. According to the approach given by Gordon and Taivassalo [7], by using a magnetic potential and the measured $B_{z}$ on the median plane at the point $(r, \theta, z)$ in cylindrical polar coordinates, the 3 rd order field can be written as

$$
\begin{aligned}
& B_{r}(r, \theta, z)=z \frac{\partial B_{z}}{\partial r}-\frac{1}{6} z^{3} C_{r}, \\
& B_{\theta}(r, \theta, z)=\frac{z}{r} \frac{\partial B_{z}}{\partial \theta}-\frac{1}{6} \frac{z^{3}}{r} C_{\theta}, \\
& B_{z}(r, \theta, z)=B_{z}-\frac{1}{2} z^{2} C_{z},
\end{aligned}
$$

where $B_{z} \equiv B_{z}(r, \theta, 0)$ and

$$
\begin{aligned}
C_{r} & =\frac{\partial^{3} B_{z}}{\partial r^{3}}+\frac{1}{r} \frac{\partial^{2} B_{z}}{\partial r^{2}}-\frac{1}{r^{2}} \frac{\partial B_{z}}{\partial r}+\frac{1}{r^{2}} \frac{\partial^{3} B_{z}}{\partial r \partial \theta^{2}}-2 \frac{1}{r^{3}} \frac{\partial^{2} B_{z}}{\partial \theta^{2}}, \\
C_{\theta} & =\frac{1}{r} \frac{\partial^{2} B_{z}}{\partial r \partial \theta}+\frac{\partial^{3} B_{z}}{\partial r^{2} \partial \theta}+\frac{1}{r^{2}} \frac{\partial^{3} B_{z}}{\partial \theta^{3}}, \\
C_{z} & =\frac{1}{r} \frac{\partial B_{z}}{\partial r}+\frac{\partial^{2} B_{z}}{\partial r^{2}}+\frac{1}{r^{2}} \frac{\partial^{2} B_{z}}{\partial \theta^{2}} .
\end{aligned}
$$

All the partial differential coefficients are computed on the median plane data by interpolation, using Lagrange's five-point formula.

The space charge fields are obtained by a quasistatic approximation. In this approach, the relative motion of the particles is nonrelativistic in the beam rest frame, so the self-induced magnetic field is practically absent and the electric field can be computed by solving Poisson's equation. The evolution of the beam's distribution function $f(\mathbf{x}, c \boldsymbol{\beta}, t)$ can be expressed by a collisionless Vlasov equation, Eq. (5). The combination of the Vlasov equation and Poisson's equation constitutes the Vlasov-Poisson system:

$$
\frac{d f}{d t}=\partial_{t} f+c \boldsymbol{\beta} \cdot \nabla_{x} f+q(\mathbf{E}+c \boldsymbol{\beta} \times \mathbf{B}) \cdot \nabla_{c \boldsymbol{\beta}} f=0 .
$$

The FFT based particle-in-cell method is used as the space charge solver. The Poisson equation is solved in the beam's rest frame $\mathbf{S}_{\text {beam }}$. In a 3D Cartesian frame, the solution of the Poisson equation at point $(x, y, z)$ can be expressed by

$$
\phi(x, y, z)=\frac{1}{4 \pi \varepsilon_{0}} \int G\left(x, x^{\prime}, y, y^{\prime}, z, z^{\prime}\right) \rho\left(x^{\prime}, y^{\prime}, z^{\prime}\right) d x^{\prime} d y^{\prime} d z^{\prime},
$$

with $G$ the 3D Green function

$$
G\left(x, x^{\prime}, y, y^{\prime}, z, z^{\prime}\right)=\frac{1}{\sqrt{\left(x-x^{\prime}\right)^{2}+\left(y-y^{\prime}\right)^{2}+\left(z-z^{\prime}\right)^{2}}},
$$

assuming open boundary conditions. We interpolate $\rho$ and $G$ onto a rectangular grid, followed by a Fourier transform 


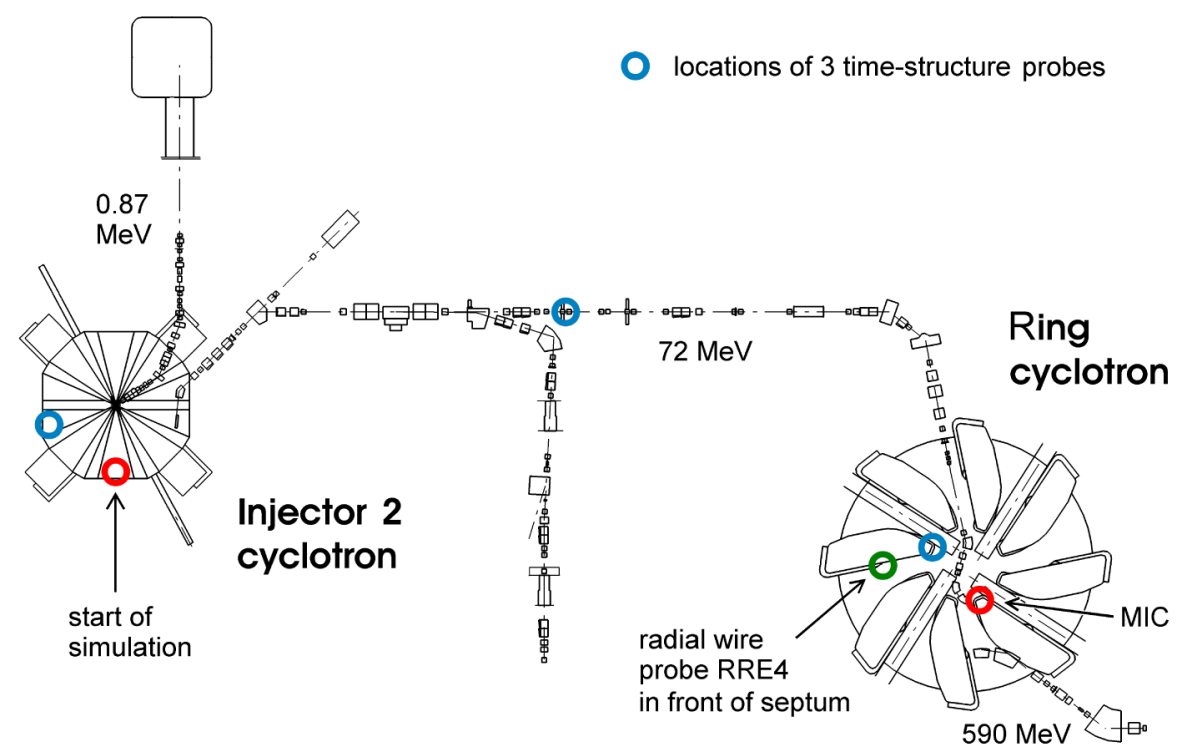

FIG. 1. The PSI Injector 2 cyclotron and beam transfer line to the PSI Ring cyclotron.

to obtain $\hat{\rho}$ and $\hat{G}$. Determining $\hat{\phi}=\hat{\rho} * \hat{G}$ and transforming them back gives $\phi=\operatorname{FFT}(\hat{\phi})^{-1}$. Still on the grid, we compute $\mathbf{E}=-\nabla \phi$ and interpolate $\mathbf{E}(\mathbf{x})$ at the particle positions $\mathbf{x}$ from $\mathbf{E}$, and finally the last step involves a Lorentz transform back to the lab frame in order to obtain $\mathbf{E}_{\mathrm{sc}}$ and $\mathbf{B}_{\mathrm{sc}}$.

\section{Computational considerations}

Both the external fields and space charge fields are used to track particles for one time step using a 4th order RungeKutta integrator, in which the fields are evaluated for 4 times in each time step. Space charge fields are assumed to be constant during one time step, because their variation is typically much slower than that of external fields. More details and unique features can be found in [6].

In the reported studies, we used between 32-256 cores, depending on the used number of particles and grid points. The running time of the simulations are in the order of 24 hours. The space charge calculations make about $50 \%$ of the total time, followed by $40 \%$ for the time integration, and $5 \%$ for the frame transformation.

\section{OBTAINING INITIAL CONDITIONS FOR THE RING CYCLOTRON}

At the extraction region of Injector 2, we only have a very limited number of measurement data; however, in the injecting beam line connecting the two cyclotrons we have 14 vertical and 17 horizontal beam profile monitors available for high intensity operation. Three time-structure measurements, one at the last turn of Injector 2, one 27 meters downstream, and one at the first turn of the PSI Ring cyclotron, give important information on the longitudinal beam size [8]. In an overview (Fig. 1) the starting point of the simulations and some of the diagnostics are shown. We note that, from a beam dynamics point of view, the particles travel in the order of $4 \mathrm{~km}$, from the marked start of the simulation to the RRE4, the probe covering the last nine turns of the PSI Ring cyclotron.

We start the OPAL-t simulations from the middle of the last valley before extraction from Injector 2 and perform a full 3D simulation until the magnetic injection channel (MIC) of the PSI Ring cyclotron. In Table I the initial values for the simulation of important beam parameters are shown. At the MIC we resample the distribution and switch to OPAL-cycl for the PSI Ring cyclotron simulation. The new distribution is sampled using the moments obtained from the transfer line simulation (at MIC).

Figure 2 shows the comparison of the $2 \sigma$ beam width between OPAL-t and the measurements. The model predicts very well the evolution of the envelope from the beginning to the end of the transfer line. Figure 3 shows the comparison of the predicted bunch length by the model and the measurements using the time-structure probes.

TABLE I. Initial conditions of the $72 \mathrm{MeV}$ transfer line for a $2 \mathrm{~mA} \mathrm{cw}$ beam. The emittances are non-normalized. The term $\left\langle x x^{\prime}\right\rangle$ is the correlation coefficient which is defined as $\left\langle x x^{\prime}\right\rangle=\frac{C\left(x, x^{\prime}\right)}{\sqrt{\left(C(x, x) C\left(x^{\prime}, x^{\prime}\right)\right.}}$, where $C\left(x, x^{\prime}\right)$ is the covariance of $x$ and $x^{\prime}$. It is the same for $\left\langle y y^{\prime}\right\rangle$ and $\langle x \delta\rangle$.

\begin{tabular}{|c|c|c|c|c|c|c|c|c|c|}
\hline Distribution & $\varepsilon_{x}(\mathrm{~mm} \mathrm{mrad})$ & $\varepsilon_{y}(\mathrm{~mm} \mathrm{mrad})$ & $x_{\mathrm{rms}}(\mathrm{mm})$ & $y_{\mathrm{rms}}(\mathrm{mm})$ & $l_{\mathrm{rms}}(\mathrm{mm})$ & $\delta(\%)$ & $\left\langle x x^{\prime}\right\rangle$ & $\left\langle y y^{\prime}\right\rangle$ & $\langle x \delta\rangle$ \\
\hline 3D Gaussian & 2.22 & 0.43 & 2.9 & 0.5 & 6.2 & 0.06 & -0.14 & 0.07 & -0.92 \\
\hline
\end{tabular}



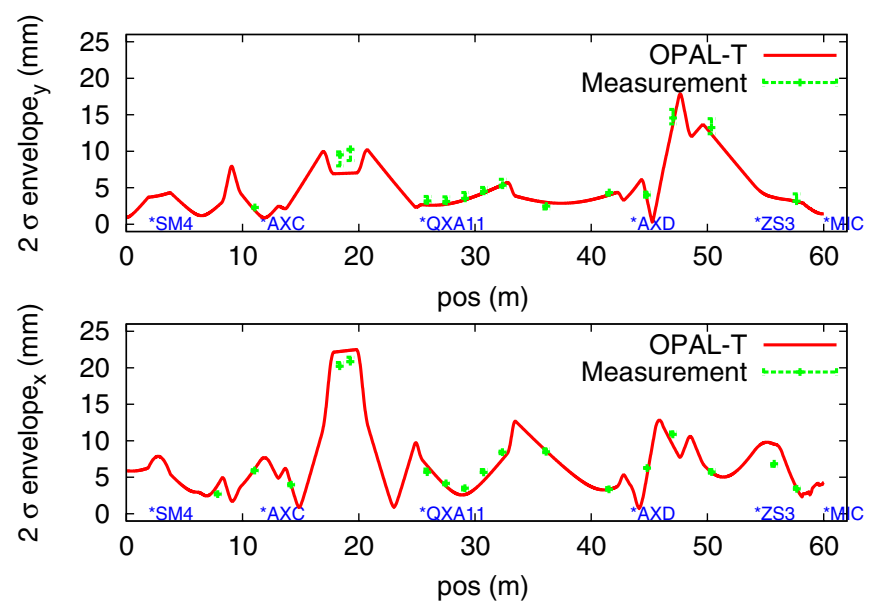

FIG. 2. Envelope of the beam in the $72 \mathrm{MeV}$ transfer line for a $2 \mathrm{~mA}$ beam. The error bars show different measurements at the same intensity level.

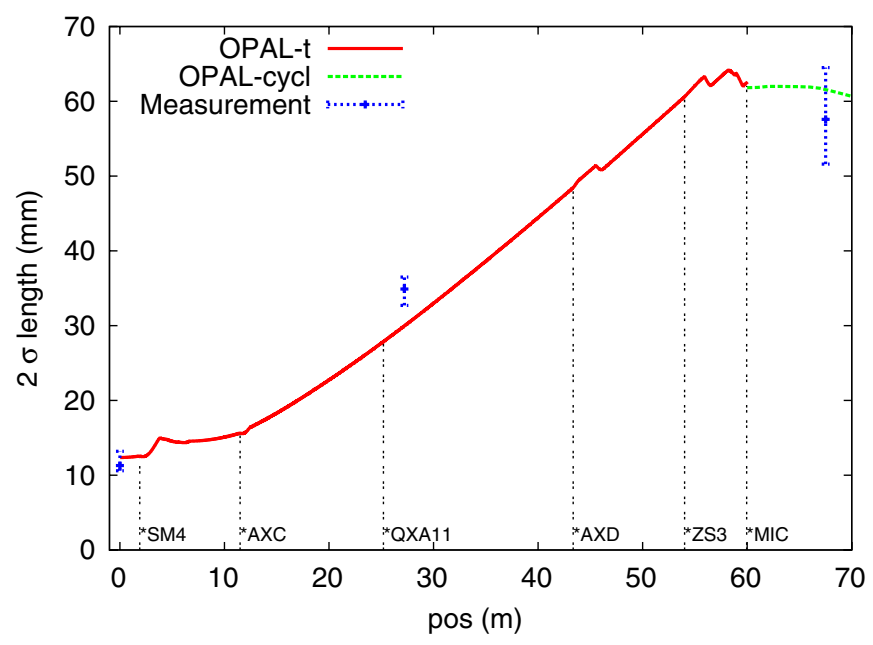

FIG. 3. Bunch length of the beam in the $72 \mathrm{MeV}$ transfer line for a $2 \mathrm{~mA}$ beam (in Fig. 1 the positions of the time-structure measurements are shown).

The large error bar at the injection to the Ring is because of the large background. The longitudinal initial conditions, in the center of the valley between sector magnet 3 and 4 (see Fig. 1), are derived from the time-structure measurement, which is $\sim 4 \mathrm{~m}$ upstream. In the center of the valley, the major axis of the bunch ellipse is along the longitudinal direction, and hence we obtain the transformed initial condition by a simple rotation in the longitudinal and radial plane.

The rf cavity fields are calculated from a normalized radial voltage profile $V(r)$, which is multiplied by the actual voltage read from the control system.

\section{A. Sensitivity}

Our goal is to minimize free parameters and also follow as much as possible the procedures developed from the operational group. Concretely, this means we are using the cavity voltages given by the control system, adjusting the initial radius, momenta, and the trim coil current of TC15 in order to (i) obtain the same amount of turns we read from the control system (error \pm 1 turn) and (ii) maximize the turn separation at extraction. In consequence, the procedure in setting up the simulations is very similar to the procedures to run the real PSI Ring cyclotron. We conclude that our sensitivity with respect to the magnetic field is in the order of a few Gauss, as seen from Figs. 4 and 7 (cf. IV B).

\section{B. Measurement considerations}

The beam horizontal and vertical profiles (projections) are measured with wire monitors. The $40 \mu \mathrm{m}$ diameter molybdenum wires (at some locations $33 \mu \mathrm{m}$ diameter carbon fibers) are mounted on forks and perform a pendulum movement with the pendulum axis $305 \mathrm{~mm}$ above or besides the beam axis and parallel to it [9]. The absolute accuracy is in the order of $1 \%-5 \%$ of the actual value for currents above $100 \mathrm{pA}$.

In the PSI Ring cyclotron, the radial turn profile (projection) is measured with a vertically stretched wire. The $33 \mu \mathrm{m}$ diameter carbon fiber is mounted on a fork and performs a linear radial movement. The secondary emission current from the wire is measured again by a logarithmic amplifier in the range from $0.1 \mathrm{nA}$ to several $\mathrm{mA}$. (The "arbitrary unit" in Figs. 7 and 8 corresponds to $10 \mathrm{nA}$ of signal current.)

The secondary emission is known to be linear over a very large dynamic range. Nevertheless, since it is a surface effect, the yield may vary over the wire surface due to varying irradiation history over the surface. We estimate the error in the yield something like a $10 \%$ effect and practically less, since the beam spot does not move much vertically (i.e., along the wire).

The detailed shape of the measured curve varies with the setup of the machine. For a well-tuned machine and simulation, repetitions of the experiment results in a very good reproducibility.

\section{TOWARDS REALISTIC HIGH POWER CYCLOTRON SIMULATIONS}

The beam losses during the operation of the cyclotron usually limit the intensity that can be extracted. The PSI $590 \mathrm{MeV}$ Ring routinely delivers $2.2 \mathrm{~mA}$ of $\mathrm{cw}$ beam, having a very low integrated loss rate, of the order of $0.02 \%$. This tight margin avoids excessive activation of accelerator components and, hence, keeps the radiation dose imposed on the personnel involved in maintenance at acceptable levels. Furthermore, about $90 \%$ of the losses are located at injection and extraction.

Therefore, the understanding of the beam dynamics and the knowledge of the detailed beam distribution especially at the extraction region, is one of the key points to be 
addressed especially if power levels increase in future projects [2].

Several important effects which need to be carefully modeled to keep extraction losses in the order of $0.02 \%$ are (i) the turn separation at the position of the extraction septum must be made as large as possible, (ii) the radial beam size at the extraction region must be smaller than the turn separation, (iii) the halo, especially at the extraction, has to be minimized, (iv) in case of the PSI Ring cyclotron, a long "pencil" beam is used and hence the linear space charge effects must be effectively compensated to avoid the formation of a S-shaped beam which apparently increases the effective radial beam size.

We now discuss these issues related to the PSI Ring cyclotron which however can be considered universal for high power cyclotrons, and hence are certainly important for future high intensity related projects [2].

\section{A. The flattop phase}

Although a compact beam is observed at the extraction of the Injector 2 cyclotron, the bunch length increases from about $\sigma=6 \mathrm{~mm}$ to about $\sigma=31 \mathrm{~mm}$ at injection into the Ring after passing through the almost $60 \mathrm{~m}$ long ( $72 \mathrm{MeV}$ ) transfer line. For such a long pencil beam, a flattop cavity is needed to compensate the energy difference from the main cavity and avoid the formation of the S-shape beam caused by space charge effects.

The flattop cavity operates at the third harmonic of the accelerating rf voltage. The energy gain per turn for any particle is the sum of four main cavities and the third harmonic cavity. When there is no space charge effect, the ideal flattop makes the total energy gain of any particle almost the same independent of the rf phase. Considering a high current beam, the flattop phase must be shifted such that the tail particles gain more energy than the head particles. This compensates exactly the linear part of the space charge force. Therefore the phase of the flattop is adjusted intensity dependent and there exists an optimum flattop phase for a given intensity.

For our simulation we use $11.5 \%$ of the sum of the main cavity voltages as the flattop cavity voltage (as set in the control room) and adjust the phase to obtain the same phase difference between main and flattop cavity as set in the control room.

\section{B. The effect of the trim coil TC15}

A small manufacturing error produces a slight deviation in the average field profile and a corresponding shift in the tunes $\nu_{r}$ and $\nu_{z}$. This requires a strong excitation of the trim coil TC15. Without this correction, the coupling resonance $\nu_{r}=2 \nu_{z}$ would be crossed 4 times at energies of $490,525,535$, and $585 \mathrm{MeV}$, respectively. A large horizontal oscillation would be transformed into a large vertical one at the coupling resonance which can lead to large vertical beam losses. An analytic model was developed

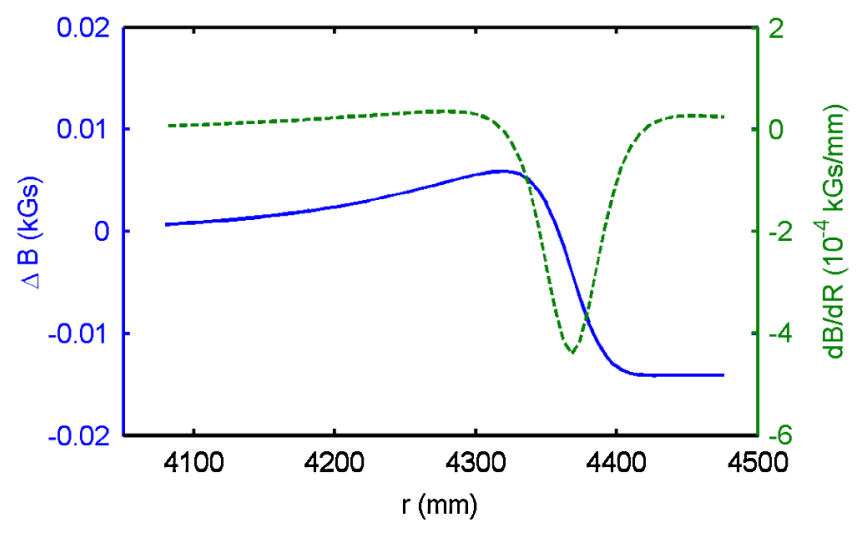

FIG. 4. The field (solid line) and field gradient (dashed line) of TC15 in the PSI Ring cyclotron.

which mimics the field due to real trim coil characteristics [10]. It is described by Eq. (8):

$$
\Delta B=-B_{a}\left[A_{1}+\frac{A_{2}}{10^{a_{1} R+b_{1}}+1}+\frac{A_{3}}{10^{a_{2} R+b_{2}}+1}\right],
$$

where $R$ is the radius, $B_{a}$ is the maximum magnetic field, the constants $A_{1}=-1.08, A_{2}=1.08, A_{3}=1.80, a_{1}=$ $0.005, b_{1}=-21.72, a_{2}=-0.033$, and $b_{2}=145.60$ for our case. It provides an additional magnet field and field gradient in the radial direction as shown in Fig. 4.

The trim coil gives a maximum magnetic field of 14 Gauss, and furthermore has a long tail towards smaller radii in order to make the integrated strength of the trim coil over the radius to zero. The radial and vertical tune shifts caused by TC15 are given by

$$
\begin{aligned}
& \Delta \nu_{r} \approx \frac{R}{2 \nu_{r} B} \frac{d \bar{B}}{d R} \approx 0.014 \\
& \Delta \nu_{z} \approx-\frac{\nu_{r}}{\nu_{z}} \Delta \nu_{r} \approx-2 \Delta \nu_{r} \approx-0.028,
\end{aligned}
$$

where $R$ is the orbit radius, $B$ is the hill field, and $\frac{d \bar{B}}{d R}$ is the average field gradient in radial direction. Careful beam dynamics studies have shown the meaningfulness of such detailed modeling in order to obtain a complete

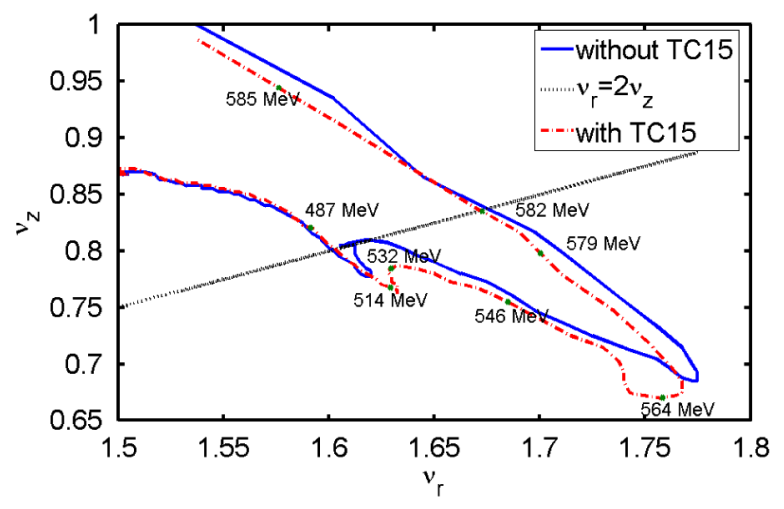

FIG. 5. Tune diagram with and without TC15. 
and precise picture of the beam dynamics in the PSI Ring cyclotron. The modified tune diagram by TC15 is shown in Fig. 5. Without TC15, in simulations and in the operation of the Ring, we observe severe vertical beam losses and cannot obtain the required extraction efficiency.

\section{The injection position and angle}

The PSI Ring cyclotron has a single turn extraction, hence a large radial turn separation between the last two turns is required. The turn separation for a centered beam is defined as

$$
\frac{d R}{d n}=\frac{\gamma}{\gamma+1} R \frac{d E / d n}{E} \frac{1}{1+k},
$$

where $k$ is the field index. For the PSI Ring cyclotron this gives about $6.0 \mathrm{~mm}$ (Fig. 6 upper part) at the extraction region, which is not enough for high current operation and would result in large losses.

To increase the turn separation, a noncentered injection into the PSI Ring cyclotron is used. Since $\nu_{r} \approx 1.7$ at extraction, adjusting the injection position and angle results in the betatron amplitude being almost equal to the increase in radius per turn. The formation of the turn pattern under this condition, for the last nine turns, is shown in Fig. 6 (lower part). This is a special turn pattern because the last turn is well separated from the overlapping second, third, and fourth last turns. In this case, the turn separation at the extraction turn is as large as $16 \mathrm{~mm}$; hence, it allows the extraction of a high intensity beam with very low losses.

\section{Comparing the radial intensity profile at extraction with measurements}

Up to now we have described the most important steps in setting up a precise beam dynamics simulation of the PSI

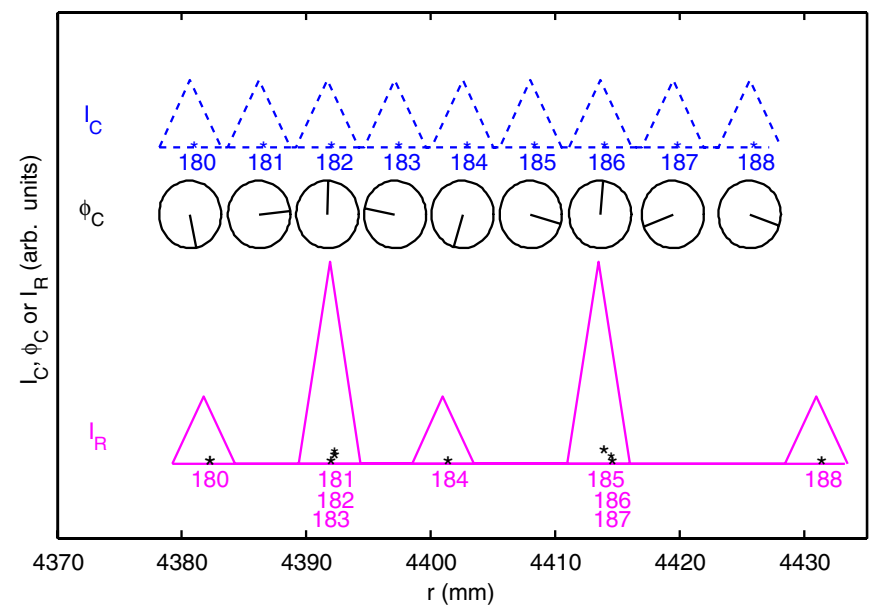

FIG. 6. Schematic representation of the turn pattern in the PSI Ring cyclotron. $I_{c}, \phi_{c}$, and $I_{r}$ represent the intensity distribution of a centered beam with $6 \mathrm{~mm}$ turn separation, the betatron oscillation phase of an eccentric beam, and the intensity distribution of a real beam with eccentric injection.
Ring cyclotron. We now compare simulations with measurements from a radial probe (RRE4) covering the last nine turns of the PSI Ring cyclotron. The probe is located $30 \mathrm{~cm}$ upstream from the $50 \mu \mathrm{m}$ thick electrostatic extraction septum and, hence, gives a very good picture of the beam distribution at the septum.

This probe is able to measure at the full intensity of the 1.3 MW cw beam. In order to compare the simulations with measurements, not only a radial probe is implemented in OPAL-cycl but also all other parameters, described in the previous sections, can be entered into the simulation.

The flattop phase and the injection position and angle are optimized to get the largest turn separation and smallest beam size at the extraction region, in both the simulation and operation of the PSI Ring cyclotron.

The effect of the trim coil TC15 on the turn pattern is shown in Fig. 7, black denotes the measurement and the colors distinguishing simulations with and without TC15. For a fixed energy the shift is given by $\frac{\Delta R}{R}=-\frac{\Delta B}{B}$. For turn 180 this shift is $\left.\Delta R\right|_{\max } \approx 3 \mathrm{~mm}$; hence, the center of turn 180 moves to the exact position of the measurement when considering the effect of the TC15. In the PSI cyclotron facility, the beam is heavily collimated during the early stage of acceleration in Injector 2 and in the beam transfer line to the PSI Ring cyclotron. As a consequence, the beam profiles do not follow a Gaussian distribution. In Fig. 8 we show again the intensity pattern at the last nine turns but for different starting distributions at MIC. Using the properties of a binomial distribution [11], we can vary a single parameter $m$ from 0 to $\infty$ and cover a wide range of distributions: from KV to Gaussian. We find that a parabolic distribution $(m=2)$ matches best the measurement at the crucial point, the septum. On the extreme side, as expected, the Gaussian distribution (truncated at $3 \sigma$ ) with its tails would fill up the intensity dip and, hence, would increase the losses at the septum. This indicated that there

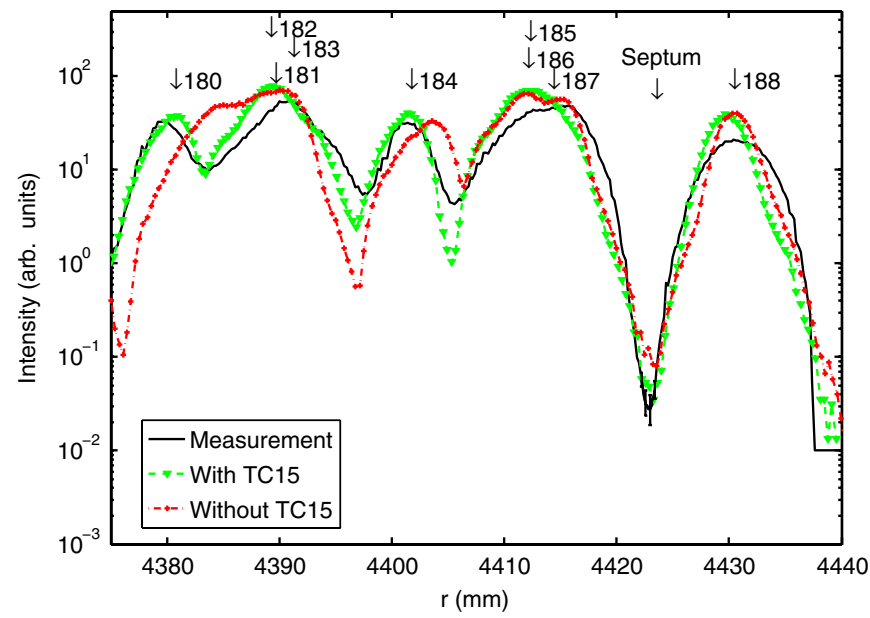

FIG. 7. Radial beam profile with indicated turn numbers at extraction for a $2 \mathrm{~mA}$ beam, for a parabolic initial distribution at MIC. 


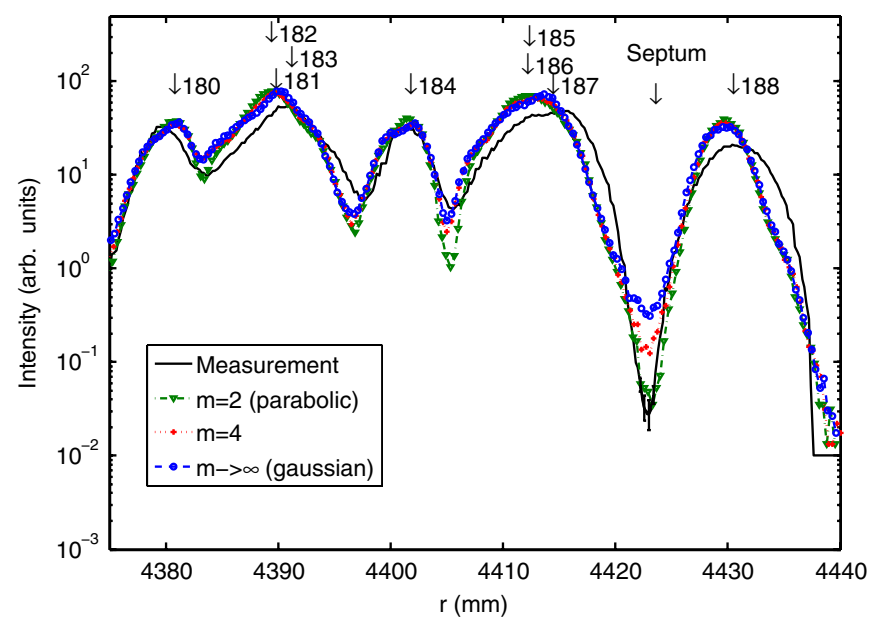

FIG. 8. The comparison of different initial beam distributions at the extraction probe, for a $2 \mathrm{~mA}$ beam.

are indeed sharp edges in the real distribution, which still differs from the assumed idealized distribution, as suggested by the remaining differences between simulation and measurement (Fig. 8, e.g., at $R=4434 \mathrm{~mm}$ ).

Nevertheless, this remarkable agreement is obtained after $\approx 4 \mathrm{~km}$ of tracking the millions of macroparticles through external fields and self-fields. This is only possible because of the parallel nature of OPAL which allows such simulations to be carried out on large high performance computing clusters. The statistical error of the measurement is indicated at $R=4423 \mathrm{~mm}$ in Figs. 7 and 8. At other radii the error bars are significantly smaller and, hence, not shown in the figures. The statistical errors of the simulation are smaller than those of the measurement errors due to the large number of simulation particles.

\section{E. SCALING LAW OF BEAM SIZE WITH RESPECT TO CURRENT}

The energy spread $\Delta E_{\mathrm{sc}}$ (linear) $=e \Delta U_{\mathrm{sc}}$ (linear) caused by the linear longitudinal space charge force after $n$ revolutions is given by Joho [12] using the sector model:

$$
\Delta U_{\mathrm{sc}} \approx Z_{I} \frac{\langle I\rangle}{\Delta \phi /(2 \pi)} \frac{n^{2}}{\beta_{f}} \text { with } Z_{I}=2.8 k \Omega=g_{1 c} \frac{64 \pi}{3} Z_{0},
$$

where $g_{1 c} \approx 1.4$ (form factor), $Z_{0}=1 / 4 \pi \varepsilon_{0} c=30 \Omega$ and $\langle I\rangle$ is the average current, $\Delta \phi$ is the phase width, $n$ the turn number and $\beta_{f}=v_{f} / c$, where $v_{f}$ is the final velocity of the beam.

The linear energy spread can be compensated with a tilted flattop voltage. This reduces the energy of leading particles and increases the energy of trailing particles. There remains a nonlinear part of the energy spread $\Delta E_{\mathrm{sc}}$ (nonlinear), which cannot be compensated. Let us define

$$
\Delta E_{\mathrm{sc}}(\text { nonlinear }) \equiv f_{n} \Delta E_{\mathrm{sc}}(\text { linear })
$$

and note that $f_{n}$ depends strongly on the beam distribution and is in our case an open parameter in the range of $0.1-0.5$.

According to Eq. (10), the space charge induced energy spread leads to a radial spread $\left(\Delta R_{\mathrm{sc}}\right)$ that results in an increase in beam size. In Fig. 9 we compare beam sizes at the extraction for beam currents from $10 \mu \mathrm{A}$ to $2.2 \mathrm{~mA}$ with simulations. Even though the measurements where done over a time span of four years with very different machine configurations, we obtain a good agreement between the simulations (theory) and the measurements. Hence, we can predict very well the extracted beam size as a function of the intensity. This simple model and the precise simulations shown in the previous paragraph constitutes a benchmarked model for the prediction of the most delicate parameters in high intensity cyclotrons.

The relation between the average energy gain $d E / d n$ and the turn number $n$ is

$$
n \frac{d E}{d n}=E_{f}-E_{i}
$$

where $E_{f}$ is the final energy and $E_{i}$ is the initial energy. For single turn extraction, the loss on the septum is limited by the ratio

$$
\frac{\Delta E_{\mathrm{sc}} \text { (nonlinear) }}{\frac{d E}{d n}}
$$

leading to the condition

$$
\Delta E_{\mathrm{sc}}(\text { nonlinear })<\mu_{n} \frac{d E}{d n} .
$$

We obtain empirically, for a centered beam a value for $\mu_{n}$ which is $\mu_{n} \approx 1 / 3$, where as for an eccentric beam, the turn separation is enhanced, and hence $\mu_{n}$ can be as high as $\mu_{n} \approx 1$.

Putting (11)-(14) together, we get for the current limit from longitudinal space charge forces,

$$
\langle I\rangle_{\max }=\frac{\mu_{n}}{f_{n}} \frac{\left(U_{f}-U_{i}\right)}{Z_{I}} \frac{\beta_{f}}{n^{3}} \frac{\Delta \phi}{2 \pi},
$$

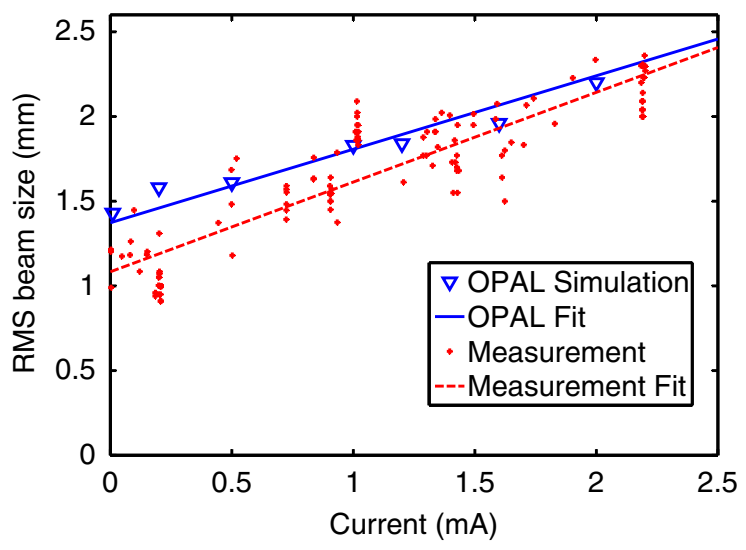

FIG. 9. Radial beam size at extraction vs beam current. 


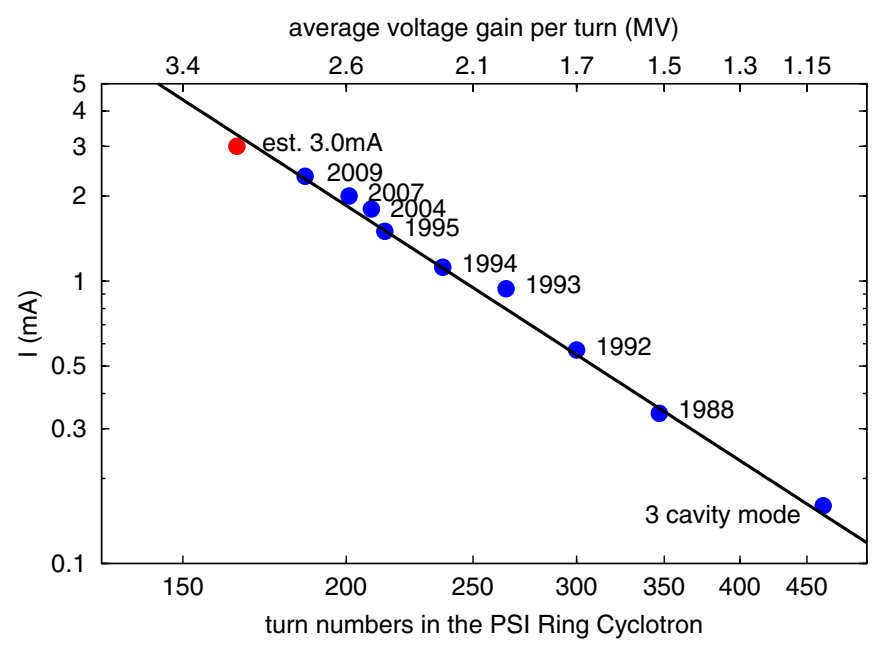

FIG. 10. Production beam current versus turn number over a span of 32 years of the PSI Ring cyclotron, in double logarithmic scale.

where $U_{f}=E_{f} / e$ and $U_{i}=E_{i} / e$. Since the turn number $n$ is inversely proportional to the cavity voltage $V_{\text {cav }}$, we see the big advantage of a large cavity voltage,

$$
\langle I\rangle_{\max } \sim \frac{1}{n^{3}} \sim V_{\text {cav }}^{3},
$$

as experimentally demonstrated by the historical current development in the PSI Ring cyclotron shown in Fig. 10.

This relation was predicted by Joho [12] in 1981, and confirmed during almost 36 years of operation of the PSI Ring cyclotron.

The prediction of the current limit in the PSI Ring cyclotron gives with (15) $\langle I\rangle_{\max }=3 \mathrm{~mA}$, using the following parameters: $U_{i}=72 \mathrm{MV}, U_{f}=590 \mathrm{MV}, \beta_{f}=0.8$, $n=188$ turns $(d E / d n=3 \mathrm{MeV}), \Delta \phi=12$ degree and estimating $f_{n}=1 / 4$ and $\mu_{n}=1$ (eccentric injection).

This is remarkably close to the present current limit of $2.3 \mathrm{~mA}$ (2010), given the crude assumptions: (i) no turn structure inside the charge sheet (see [12] Fig. 3), (ii) nonrelativistic approximation, (iii) no radial boundary condition, and (iv) uncertainties in $f_{n}$ and $\mu_{n}$.

We note that for the PSI Injector 2 (15) is not applicable due to phase mixing [13] and, hence, would give a pessimistic value for the current limit.

\section{CONCLUSIONS AND DISCUSSIONS}

In this paper, we present novel precise simulations for the beam dynamics in a high intensity cyclotron. For the first time we are able to obtain a realistic and detailed understanding of the beam dynamics in the very complex PSI Ring cyclotron by means of 3D particle simulations. By a rough estimation of the initial distribution, according to measurements of beam profile monitors, and the time structure of the beam, realistic simulations of the PSI Ring cyclotron are presented and compared to measurements.
Very good agreement for the radial probe between the simulation and measured data is obtained by adjusting the injection position, angle, flattop voltage, and the trim coil TC15. These parameters are all in agreement with settings obtained from the control room.

The presented results with a level of detail large enough to predict limiting tails on the extraction septum (at beam width levels of $3 \sigma-4 \sigma$ ), and can be seamless extrapolated to future high power cyclotrons and enable the precise prediction of crucial parameters, such as losses, based on an existing cw megawatt facility experiences. However, a crucial part is the knowledge of the initial distribution from the evaluation of measurements of beam profiles and time structure.

Primary modeling limitations include an accurate knowledge of the initial particle distribution in the full 6D phase space, and the lack of particle-matter interaction in our model. Particle-matter interaction models and resulting struggled primary particles and electrons will play an important role when intensity levels increase, while at the same time the losses must be held at present levels. We plan to include these effects in future studies; preliminary results on the particle-matter interaction model are reported in [14] and ideas for secondary electron creation and field emission can be found in [15].

\section{ACKNOWLEDGMENTS}

The authors thank the Accelerator Modeling and Advanced Simulation (AMAS) group members C. Kraus, Y. Ineichen, and J. J. Yang for many discussions regarding programming and T. Schietinger for providing the postprocessing tool H5PARTROOT. We also thank H.Zhang for providing information of the $72 \mathrm{MeV}$ injection line and the PSI Ring cyclotron. This work was partly performed on the felsim cluster at the Paul Scherrer Institut and on the Cray XT5 at Swiss National Supercomputing Center (CSCS) within the "Horizon" collaboration.

\section{APPENDIX A: DISTRIBUTION GENERATION}

Particle distributions are generated separately in all three phase space planes. There are limited correlations between planes, e.g., between longitudinal and transverse $\left(\langle x l\rangle,\left\langle x^{\prime} l\right\rangle,\langle x \delta\rangle,\left\langle x^{\prime} \delta\right\rangle\right)$. Besides an efficient parallel Gaussian distribution generator based on a parallelized "Method of Rejection," a more general algorithm for generating distributions is also used [11]. The phase space density of a truncated binomial distribution is defined as

$$
\rho(u, v)= \begin{cases}\frac{m}{\pi}\left(1-a^{2}\right)^{m-1} & \text { for } a \leq 1 \\ 0 & \text { for } a \geq 1,\end{cases}
$$

where $(u, v)$ are the dimensionless Cartesian coordinates, $a^{2}=u^{2}+v^{2}$. The connection between $(u, v)$ and the real phase space Cartesian coordinates $\left(x, x^{\prime}\right)$ is

$$
x=x_{L} \cdot u \quad x^{\prime}=x_{L}^{\prime}(u \sin \chi+v \cos \chi),
$$

where $x_{L}$ is the limiting amplitude, $x_{L}^{\prime}$ is the limiting divergence, and $\chi$ is the correlation phase for the 
TABLE II. Different distributions specified by a single parameter $m$. The symbol $\delta$ in the hollow shell distribution is the Dirac function.

\begin{tabular}{lcccc}
\hline \hline $\mathrm{m}$ & Distribution & Phase space density & Profile & $\begin{array}{c}\text { Truncation } \\
\left(x_{L} / \sigma_{x}\right)\end{array}$ \\
\hline 0.0 & Hollow shell & $\frac{1}{\pi} \delta\left(1-a^{2}\right)$ & $\frac{1}{\pi}\left(1-u^{2}\right)^{-0.5}$ & $\sqrt{2}$ \\
0.5 & Flat profile & $\frac{1}{2 \pi}\left(1-a^{2}\right)^{-0.5}$ & $\frac{1}{2}$ & $\sqrt{3}$ \\
1.0 & Uniform & $\frac{1}{\pi}$ & $\frac{2}{\pi}\left(1-u^{2}\right)^{0.5}$ & 2 \\
1.5 & Elliptical & $\frac{3}{2 \pi}\left(1-a^{2}\right)^{0.5}$ & $\frac{3}{4}\left(1-u^{2}\right)$ & $\sqrt{5}$ \\
2.0 & Parabolic & $\frac{2}{\pi}\left(1-a^{2}\right)$ & $\frac{8}{3 \pi}\left(1-u^{2}\right)^{1.5}$ & $\sqrt{6}$ \\
3.5 & & $\frac{7}{2 \pi}\left(1-a^{2}\right)^{2.5}$ & $\frac{35}{32}\left(1-u^{2}\right)^{3}$ & 3 \\
7.0 & & $\frac{7}{\pi}\left(1-a^{2}\right)^{6}$ & $1.52\left(1-u^{2}\right)^{6.5}$ & 4 \\
$\rightarrow \infty$ & Gaussian & $\frac{1}{2 \pi \sigma_{x} \sigma_{x^{\prime}}} \exp \left(-\frac{x^{2}}{2 \sigma_{x}^{2}}-\frac{x^{2}}{2 \sigma_{x^{\prime}}^{2}}\right)$ & $\frac{1}{\sqrt{2 \pi} \sigma_{x}} \exp \left(-\frac{x^{2}}{2 \sigma_{x}^{2}}\right)$ & $\infty$ \\
\hline \hline
\end{tabular}

orientation of the ellipse. The rms amplitude $\sigma_{x}$ is then given by

$$
\sigma_{x}=x_{L} \sqrt{2(m+1)}
$$

It is well known that the shape of the binomial distribution is governed by one single parameter $m$. By varying this single parameter, one obtains the most commonly used distributions in beam dynamics simulations, as listed in Table II.

[1] J. M. Conrad and M.H. Shaevitz, Phys. Rev. Lett. 104, 141802 (2010).

[2] H. A. Abderrahim et al., DOE Technical Report (2010), Accelerator and Target Technology for Accelerator Driven Transmutation and Energy Production.
[3] A. Adelmann, Y. Bi, C. Kraus, Y. Ineichen, S. Russel, and J. Yang, Paul Scherrer Institut Technical Report No. PSIPR-08-02, 2008.

[4] A. Adelmann, Paul Scherrer Institut Technical Report No. PSI-PR-09-05, 2009.

[5] A. Adelmann, P. Arbenz, and Y. Ineichen, J. Comput. Phys. 229, 4554 (2010) [http://www.science direct.com/science/article/B6WHY-4YHP08T-1/2/41309 c23eb7fa1b4af95d9401a21da39].

[6] J. J. Yang, A. Adelmann, M. Humbel, M. Seidel, and T. J. Zhang, Phys. Rev. ST Accel. Beams 13, 064201 (2010).

[7] M. M. Gordon and V. Taivassalo, IEEE Trans. Nucl. Sci. 32, 2447 (1985).

[8] R. Dölling, in Proceedings of HB2010 (Paul Scherrer Institut, Morschach, Switzerland, 2010).

[9] L. Rezzonico, in Proceedings of the 14th International Conference on Cyclotrons and their Applications (World Scientific, Cape Town, South Africa, 1996), p. 457.

[10] S. Adam and W. Joho, PSI Technical Report No. TM-1113, 1974.

[11] W. Joho, PSI Technical Report No. TM-11-14, 1980.

[12] W. Joho, in the 9th International Conference on Cyclotrons (Editions de Physique, Caen, France, 1981), p. 337.

[13] S. Adam, IEEE Trans. Nucl. Sci. 32, 2507 (1985).

[14] Y. J. Bi, A. Adelmann, R. Dölling, W. Joho, M. Seidel, C. X. Tang, and T. J. Zhang, in Proceedings of HB2010 (Ref. [8]).

[15] C. Wang, A. Adelmann, and Y. Ineichen, in Proceedings of HB2010 (Ref. [8]). 